Series A

\author{
I. MATHEMATICA
}

384

\title{
ON LOCAL ASYMPTOTIC PROPERTIES, THE ASYMPTOTIC VALUE SETS, AND AMBIGUOUS PROPERTIES OF FUNCTIONS MEROMORPHIC IN THE OPEN UNIT DISC
}

J. E. McMILLAN 
Communicated 8 October 1965 by Olli Lehto and K. I. Virtanen 


\section{Introduction}

We first prove a theorem (Theorem 1) that generalizes a classical theorem of Gross and Iversen (see [6, p. 124]) and a recent theorem of MacLane [7, Theorem 11]. It is closely related to the Collingwood-Cartwright main theorem in the small [6, Theorem 16], and it is a local relative of a global theorem in [9, Theorem 2]. In Section 3 we generalize the result of Mazurkiewicz [8] that states that the asymptotic value set of a function meromorphic in the disc is an analytic set. We prove, roughly speaking, that for functions meromorphic in the disc, the asymptotic image of an analytic set (in the circumference) is an analytic set. This result is applied in the proof of Theorem 1. In Section 4 we apply Theorem 1 to the study of ambiguous properties, and prove that if a holomorphic function has no ambiguous points, then it must have a principal cluster value at each point of a residual set.

Let $f$ be a function meromorphic in the open unit disc $D:|z|<1$, let $C$ denote the circumference of $D$, and let $\Omega$ denote the extended plane. We use the chordal metric $d(a, b)$ on $\Omega$, and let $N(a, \varepsilon)$ and $N^{\prime}(a, \varepsilon)$ $(a \in \Omega, \varepsilon>0)$ denote the sets $\{w: d(a, w)<\varepsilon\}$ and $\{w: 0<d(a, w)<\varepsilon\}$, respectively. We say that a simple curve $\beta: z(t)(0 \leq t<1)$ contained in $D$ is a boundary curve provided $|z(t)| \rightarrow 1$ as $t \rightarrow 1$, and we say that $f$ has the limit $a(a \in \Omega)$ on $\beta$ if $f(z(t)) \rightarrow a$ as $t \rightarrow 1$. The set $\bar{\beta} \cap C$ (the bar denotes closure) is called the end of $\beta$. We call a boundary curve with end the point $\zeta$ of $C$ an arc at $\zeta$. We say that $f$ has the asymptotic value $a$ in the set $S(S \subset C)$ if $f$ has the limit $a$ on a boundary curve that has end contained in $S$, and that $f$ has the asymptotic value a at $\zeta(\zeta \in C)$ if there is an arc at $\zeta$ on which $f$ has the limit $a$. Denote the set of all asymptotic values of $f$ in $S(S \subset C)$ by $\Gamma(S)$ and the set of all asymptotic values of $f$ at points of $S$ by $\Gamma_{p}(S)$. For a set $S \subset \Omega$, let $A(S)$ denote the set $\{\zeta \in C$ : there exists $a \in S$ such that $f$ has the asymptotic value $a$ at $\zeta\}$, and let $A=A(\Omega)$. We say that a closed arc $\gamma$ of $C$ with endpoints $\zeta_{1}$ and $\zeta_{2}$ is a Koebe arc of $f$ for the value $a(a \in \Omega)$ if to each positive number $\varepsilon$ there corresponds a Jordan arc $\gamma_{\varepsilon}$ contained in $\{1-\varepsilon<|z|<1\}$, having distance less than $\varepsilon$ from both $\zeta_{1}$ and $\zeta_{2}$, and on which $d(f(z), a)<\varepsilon\left(z \in \gamma_{\varepsilon}\right)$. 


\section{Local asymptotic properties}

Theorem 1. Suppose that the value $a(a \in \Omega)$ is not assumed by $f$ arbitrarily near $\zeta(\zeta \in C)$, and that there exists a sequence $\left\{z_{n}\right\} \subset D$ such that $z_{n} \rightarrow \zeta$ and $f\left(z_{n}\right) \rightarrow a$. Then at least one of the following three statements is true.

(1) $f$ has the asymptotic value $a$ in each open arc containing $\zeta$.

(2) $\zeta$ is one end of a Koebe arc of $f$ for the value $a$.

(3) For each positive number $\varepsilon$ and open arc $\gamma$ containing $\zeta$, $\gamma \cap A\left(N^{\prime}(a, \varepsilon)\right)$ has positive Lebesgue measure (in $\left.[0,2 \pi]\right)$, and $N(a, \varepsilon) \cap \Gamma_{p}(\gamma)$ has positive linear measure.

(See the end of Section 3 for the definition of linear measure.)

Remark 1. A special case of the Collingwood-Cartwright main theorem in the small [6, Theorem 16] is the inclusion (in their notation)

$$
[\Omega-R(f, \zeta)] \cap C(f, \zeta) \subset \chi^{*}(f, \zeta) \cup \Phi(f, \zeta),
$$

which generalizes the classical theorem of Gross and Iversen (see [6, p. 124]). It is clear that Theorem 1 is a refinement of this inclusion.

Remark 2. Since a function meromorphic and normal in $D$ can have no Koebe arc [4, Theorem 1], Theorem 1 leads to an instructive proof of the theorem of Bagemihl and Seidel [4, Theorem 3] that states that if $f$ is holomorphic and normal in $D$, then for each arc $\gamma$ of $C$, either some point of $\gamma$ is a Fatou point of $f$ for which the corresponding Fatou value is $\infty$, or the set of Fatou points of $f$ on $\gamma$ has positive Lebesgue measure.

Remark 3. MacLane [7, Theorem 11] proved that if $f$ is holomorphic in $D$ and $A$ is dense on $C$, then for each arc $\gamma$ of $C$, either $\gamma \cap A(\{\infty\}) \neq \varnothing$ or $\gamma \cap A(\Omega-\{\infty\})$ has positive Lebesgue measure. It is clear that this result is implied by Theorem 1. In my thesis [9, Theorem 2] I extend MacLane's arguments and prove that if $f$ is holomorphic in $D$, has only finitely many asymptotic tracts for $\infty$, and the union of the ends of the arc-tracts of $f$ for the value $\infty$ is not all of $C$, then $A(\Omega-\{\infty\})$ has positive Lebesgue measure (for the definition of "tract» see Section 3 ). The proofs of this result and Theorem 1 are based on the same lemma [9, Lemma $2]$.

Remark 4. The linear measure part of Theorem 1 is related to theorems given by Bagemihl [2].

Proof of Theorem 1. Suppose that (1) is false. Let $\delta$ be a positive number such that $f$ does not have the asymptotic value $a$ in $C \cap\{|z-\zeta| \leq \delta\}$, and $f$ does not assume the value $a$ in $D \cap\{|z-\zeta|<2 \delta\}$. We write $U=D \cap\{|z-\zeta|<\delta\}$, and to simplify the notation, we suppose that $z_{n} \in U(n \geq 1)$. Let $\Delta_{n}$ be the component of $\left\{z: d(f(z), a)<2 d\left(f\left(z_{n}\right), a\right)\right\}$ that contains $z_{n}$. Suppose now that (2) is also false; then the diameter 
of $\Delta_{n} \cup\{\zeta\}$ tends to zero as $n \rightarrow \infty$. We wish to prove that (3) is true. Let $\varepsilon$ be a positive number, let $\gamma$ be an open are containing $\zeta$, and let $n_{0}$ be such that $\bar{\Delta}_{n_{0}} \subset U \cup \gamma$ and $2 d\left(f\left(z_{n_{0}}\right), a\right)<\varepsilon$. Let $\Delta_{0}=\Delta_{n_{0}}$ and $\delta_{0}=2 d\left(f\left(z_{n_{0}}\right), a\right)$. Then $\delta_{0}<\varepsilon, \Delta_{0}$ is a component of $f^{-1}\left(N\left(a, \delta_{0}\right)\right)$, and $\bar{\Delta}_{0} \subset U \cup \gamma$. We now prove that there exist a positive number $\delta^{\prime}\left(\delta^{\prime} \leq \delta_{0}\right)$ and some component of $f^{-1}\left(N\left(a, \delta^{\prime}\right)\right)$ that is contained in $\Delta_{0}$ and in which $f$ is bounded away from $a$. Otherwise, we could choose $\delta_{n} \downarrow 0 \quad\left(\delta_{n}<\delta_{0}\right)$, let $D_{1}$ be a component of $f^{-1}\left(N\left(a, \delta_{1}\right)\right)$ that is contained in $\Delta_{0}$, let $D_{2}$ be a component of $f^{-1}\left(N\left(a, \delta_{2}\right)\right)$ that is contained in $D_{1}$, and in this way, define a sequence $\left\{D_{n}\right\}$ such that $D_{1} \subset \Delta_{0}, D_{n+1} \subset D_{n}$, and $D_{n}$ is a component of $f^{-1}\left(N\left(a, \delta_{n}\right)\right)$. It is clear that $f$ would have the limit $a$ on a boundary curve contained eventually (for sufficiently large $|z|$ ) in each $D_{n}$, and this is not compatible with the inclusion $\Delta_{0} \subset U$. Thus, we may let $\delta^{\prime}$ and $\Delta$ be such that $\left(\delta^{\prime} \leq \delta_{0}\right) \Delta$ is a component of $f^{-1}\left(N\left(a, \delta^{\prime}\right)\right)$ that is contained in $\Delta_{0}$ and in which $f$ is bounded away from $a$. A proof in [9, Proof of Lemma 2] yields

Lemma 1. Suppose that $f$ is holomorphic in $D$, and that $f$ does not have the asymptotic value $\infty$. Suppose that $\Delta$ is a component of $\{z:|f(z)|>\lambda\}$ in which $f$ is bounded. Then there exists a set $E \subset C$ of positive exterior Lebesgue measure such that there is an arc at each $e^{i \Theta} \in E$ that is contained in $\Delta$ and on which $f$ has a limit at $e^{i \theta}$.

It is clear that we can apply Lemma 1 to the restriction of the function $\frac{1}{f-a}$ to $U$ to obtain that the set $\gamma \cap A\left(N^{\prime}(a, \varepsilon)\right)$ has positive exterior Lebesgue measure. Thus, since the measurability of $A\left(N^{\prime}(a, \varepsilon)\right)$ follows from Theorem 2, Corollary (ii)', we have proved that $\gamma \cap A\left(N^{\prime}(a, \varepsilon)\right)$ has positive Lebesgue measure.

We now prove that $N(a, \varepsilon) \cap \Gamma_{p}(\gamma)$ has positive linear measure. Let $l^{*}$ denote exterior linear measure (see the end of Section 3 ). It is routine to verify that if $T(z)$ is a linear transformation of $\Omega$ and the set $R$ in $\Omega$ satisfies $l^{*}(R)>0$, then $l^{*}(T(R))>0$. Thus, since the linear measurability of $\Gamma_{p}(\gamma)$ follows from Theorem 2, Corollary (i)', it is sufficient to prove that $l^{*}\left(N(a, \varepsilon) \cap \Gamma_{p}(\gamma)\right)>0$ under the assumption that $a=\infty$. We may clearly suppose that $f$ assumes a positive real value in $\Delta_{0}$, for otherwise we could arrive at this case by a rotation of $\Omega$. We suppose then that $a=\infty$ and that the positive real number $u_{0}$ is assumed by $f$ at some point of $\Delta_{0}$. Let $h$ be a positive number such that if we write $L=\{u+i v$ : $\left.u=u_{0},|v|<h\right\}$, then $L$ has a lifting $L^{*}$ ontc the Riemann surface $s$ of $f$, and $L^{*}$ corresponds under $f$ to a curve in $\Delta_{0}$. Since $f$ does not have the asymptotic value $\infty$ in $\bar{U} \cap C$ and $f$ is holomorphic in $\Delta_{0}$, for each real number $v$ satisfying $|v|<h$, there is a largest real number $u_{v}$ 
with the property that $\left\{u+i v: u_{0} \leq u<u_{v}\right\}$ has a lifting onto $s$ beginning at the point of $L^{*}$ over $u_{0}+i v$. It follows that each $u_{v}+i v$ $(|v|<h)$ is an asymptotic value of $f$ in the arc $\gamma$ and must, because of the choice of $\Delta_{0}$, lie in $N^{\prime}\left(\infty, \delta_{0}\right)$. We now write $\gamma^{*}=\{|z-\zeta| \leq \delta\} \cap C$, and prove that $f$ can have only countably many asymptotic values on boundary curves that end in subarcs of $\gamma^{*}$. If this were not the case, there would exist boundary curves $\beta_{j}(j=1,2)$ with end $\gamma_{j} \subset \gamma^{*}$ on which $f$ has the limit $w_{j}$ such that $\gamma_{1} \cap \gamma_{2} \neq \varnothing$ and $w_{1} \neq w_{2}$. We clearly may suppose that $\beta_{1} \cup \beta_{2}$ is a simple curve and that if we let $\Delta^{*}$ be the subdomain of $D$ that has $\beta_{1} \cup \beta_{2} \cup \gamma_{1} \cup \gamma_{2}$ as its boundary, then $f$ is holomorphic in $\Delta^{*}$. Since $\gamma_{1} \cap \gamma_{2} \neq \varnothing$, it follows easily from Koebe's lemma (and is obvious from the theory of prime ends) that under conformal mapping of $\Delta^{*}$ onto $D, \beta_{1} \cup \beta_{2}$ corresponds to $C$ minus a single point. Thus, from a well known theorem of Lindelöf (see [5, p. 38]), $f$ is unbounded in $\Delta^{*}$; and from a theorem of Gross and Iversen (see [6, p. 124]), the restriction of $f$ to $\Delta^{*}$ must have the asymptotic value $\infty$. That is, $f$ has the asymptotic value $\infty$ in $\gamma^{*}$, and this is not compatible with the choice of $\delta$. Thus, since $\bar{\Delta}_{0} \cap C \subset \gamma^{*}$, all but countably many cf the points $u_{v}+i v \quad(|v|<h)$ are in $N(\infty, \varepsilon) \cap \Gamma_{p}(\gamma)$; and by projection of these points onto $L$, we see that $l *\left(N(\infty, \varepsilon) \cap \Gamma_{p}(\gamma)\right)>0$. Thus, assuming the results from Section 3 , the proof of Theorem 1 is complete.

\section{The asymptotic value sets}

The main result of this section is

Theorem 2. Suppose that $f$ is meromorphic in D. Then

(i) for each analytic set $S \subset C$, both $\Gamma_{p}(S)$ and $\Gamma(S)$ are analytic sets in $\Omega$,

and

(ii) for each analytic set $S \subset \Omega, A(S)$ is an analytic set in $C$.

We first introduce the notion of an asymptotic tract of $f$ (for a discussion of asymptotic tracts, see [7, p. 5]). Let $a \in \Omega$, and suppose that to each positive number $\varepsilon$ there corresponds a component $D(\varepsilon)$ of $f^{-1}(N(a, \varepsilon))$ such that $D\left(\varepsilon_{1}\right) \subset D\left(\varepsilon_{2}\right) \quad\left(\varepsilon_{1}<\varepsilon_{2}\right)$ and $\bigcap_{\varepsilon>0} D(\varepsilon)=\varnothing$. Then we say that $\{D(\varepsilon)\}$ is an asymptotic tract (or simply tract) of $f$ for the value $a$, and call $\bigcap_{\varepsilon>0} \bar{D}(\varepsilon)$ the end of the tract. A tract is a point-tract or an arc-tract depending on whether its end is a point or an arc of $C$. We say that a boundary curve belongs to the tract $\{D(\varepsilon)\}$ if for each $\varepsilon$ it is eventually (for sufficiently large $|z|$ ) in $D(\varepsilon)$. 
We shall need

Theorem 3. With the exception of only countably many tracts of $f$, each boundary curve belonging to a tract has end equal to the end of the tract.

Remark. Theorem 3 generalizes a (rather trivial) theorem in [9, Theorem 5].

Proof of Theorem 3. Suppose that the assertion is false. Then $f$ has uncountably many tracts $T$ with the property that there exists a boundary curve $\beta(T)$ belonging to $T$ such that the end $\gamma(T)$ of $\beta(T)$ is a proper subset of the end $K(T)$ of $T$. It follows that there exist a positive number $\delta$, a nonnegative integer $n$, and an open arc $\gamma^{*}$ of $C$ with length $(n+1) \delta$ such that for uncountably many $T$ it is the case that ( $l$ denotes length) $l[K(T)]-l[\gamma(T)]>\delta, \quad n \delta \leq l[\gamma(T)]<(n+1) \delta, \quad$ and $\quad \gamma(T) \subset \gamma^{*}$. But for any three of the tracts $T$ satisfying the relation $\gamma(T) \subset \gamma^{*}$, one must satisfy $K(T) \subset \gamma^{*}$; and this contradicts the relations

$$
l[K(T)]>\delta+l[\gamma(T)] \geq \delta+n \delta=l\left[\gamma^{*}\right] .
$$

Thus the proof of Theorem 3 is complete.

The Mazurkiewicz metric (see [8]) on the Riemann surface $s$ of $f$ is defined as follows. Let $P$ and $Q$ be points of $s$, and let $\varrho(P, Q)$ denote the infimum over all Jordan ares $J$ on $s$ joining $P$ and $Q$ of the diameter (in the chordal metric) of the projection of $J$ onto $\Omega$. Then (see Mazurkiewicz [8] and Seibert [12, pp. 341-34' and footnote 5]) $\varrho$ is a metric on $s$, the completion $\left\{s^{*}, \varrho\right\}$ of $\{s, \varrho\}$ is a (complete) separable metric space, and the projection mapping on $s$ has a continuous extension $\pi$ to $s^{*}$. We write $B=s^{*}-s$.

Now let $P \in B$, and let $\left\{P_{n}\right\}$ be a sequence of points of $s$ such that $\varrho\left(P, P_{n}\right) \rightarrow 0$. For each positive number $\varepsilon$, let $\Delta(P, \varepsilon)$ be the component of $s$ over $N(\pi(P), \varepsilon)$ that contains all but finitely many of the points $P_{n}$. It is clear that $\Delta(P, \varepsilon)$ does not depend on the choice of $\left\{P_{n}\right\}$. Let $D(P, \varepsilon)$ be the subset of $D$ that corresponds under $f$ to $\Delta(P, \varepsilon)$. Then $\{D(P, \varepsilon)\}$ is an asymptotic tract of $f$ for the value $\pi(P)$, and it is clear that the correspondence $P \leftrightarrow\{D(P, \varepsilon)\}$ is one-to-one. We let $B^{P}$ denote the set of points of $B$ that correspond to point-tracts of $f$. It is clear that for each positive integer $n$, the set of points of $B$ that correspond to arc-tracts with ends having length greater than or equal to $\frac{1}{n}$ is closed in $s^{*}$. Thus, $B^{p}$ is a $G_{\delta}$ set in $s^{*}$. Let $\psi(P)\left(P \in B^{p}\right)$ denote the point of $C$ that is the end of the tract of $f$ corresponding to $P$. Then $\psi$ is continuous on $B^{p}$.

In the following proofs we make repeated use of the facts that (in $\Omega$ or $C$ ) a Borel set is an analytic set, and the union or intersection of two analytic sets is an analytic set (see [13, pp. 212-213]).

Proof of (i). Let $S$ be an analytic set in $C$. Since $\psi$ is continuous, it is 
elementary that $\psi^{-1}(S) \quad\left(=\left\{P \in B^{p}: \psi(P) \in S\right\}\right)$ is an analytic set relative to $B^{p}$. Thus, since $B^{p}$ is a $G_{\delta}$ set in $s^{*}, \psi^{-1}(S)$ is an analytic set in $s^{*}$. Now let $B^{\prime}$ be the set of points of $B$ that correspond to tracts $T$ with the property that there is an arc at some point of $S$ that belongs to $T$. It follows from Theorem 3 that $B^{\prime}$ is equal to $\psi^{-1}(S)$ plus a countable set, and is thus an analytic set in $s^{*}$. Since $B^{\prime}$ is an analytic set in the complete, separable metric $s^{*}$ and $\pi$ is a continuous mapping of $B^{\prime}$ onto $\Gamma_{p}(S)$ (which is contained in the complete, separable metric space $\Omega$ ), it follows that $\Gamma_{p}(S)$ is an analytic set (see [13, p. 219]). (Mazurkiewicz [8] used $\pi$ to prove that $\Gamma(C)$ is an analytic set.)

Now let $B^{\prime \prime}$ denote the set of points of $B$ that correspond to tracts $T$ with the property that there is a boundary curve that belongs to $T$ and has end contained in $S$; then $\pi\left(B^{\prime \prime}\right)=\Gamma(S)$. As in the argument just given, in order to show that $\Gamma(S)$ is an analytic set, it is sufficient to prove that $B^{\prime \prime}$ is an analytic set in $s^{*}$. For a set $H \subset C$, let $B_{H}$ denote the set of points of $B$ that correspond to tracts with ends contained in $H$. We now prove that if $\gamma$ is an arc of $C$, then $B_{\gamma}$ is a $G_{\delta \sigma}$ set in $s^{*}$. Since we can write any arc as the union of an increasing sequence of closed arcs, it is sufficient to prove that if $\gamma$ is a closed arc, then $B_{\gamma}$ is a $G_{\delta}$ set in $s^{*}$. Suppose then that $\gamma$ is a closed arc of $C$, and let $B(n)$ denote the set of points of $B$ that correspond to tracts with ends containing points at a distance greater than or equal to $\frac{1}{n}$ from $\gamma$. Since $\gamma$ is closed, $B_{\gamma}=B-\bigcup_{n=1}^{\infty} B(n)$. Thus, since $B$ and $B(n)(n \geq 1)$ are closed in $s^{*}, B_{\gamma}$ is a $G_{\delta}$ set.

Now let $U$ denote the interior of $S$, let $E$ denote the set of endpoints of the components of $U$, and write $H=U \cup(E \cap S)$. Since for each component $\gamma$ of $H, B_{\gamma}$ is a $G_{\delta \sigma}$ set, we see that $B_{H}$ is a $G_{\delta \sigma}$ set. Thus, since $B_{s}=B_{H} \cup \psi^{-1}(S), B_{s}$ is an analytic set in $s^{*}$; and it follows from Theorem 3 that $B^{\prime \prime}$ is equal to $B_{s}$ plus a countable set, and is thus an analytic set in $s^{*}$. Thus the proof of (i) is complete.

Proof of (ii). Let $S$ be an analytic set in $\Omega$, and let $\pi_{B}$ be the restriction of $\pi$ to $B$. Since $\pi_{B}$ is continuous, $\pi_{B}^{-1}(S)$ is an analytic set relative to $B$. Thus, since $B$ is closed in $s^{*}, \pi_{B}^{-1}(S)$ is an analytic set in $s^{*}$, and $B^{p} \cap \pi_{B}^{-1}(S)$ is an analytic set in $s^{*}$. Thus, as before, $\psi\left(B^{p} \cap \pi_{B}^{-1}(S)\right)$ is an analytic set in $C$. (We note that we neither need nor have that $\psi$ has a continuous extension to the complete space.) That is, the set $A^{\prime}$ of ends of point-tracts for values in $S$ is an analytic set. Since the end of an arc-tract is the end of a boundary curve belonging to the tract, it is easy to see that for a given tract of $f$, the set of points of $C$ that are ends of arcs belonging to that tract is closed. Thus it follows from Theorem 3 that $A(S)$ is equal to $A^{\prime}$ plus a countable union of closed sets, and is thus an analytic set; and the proof of (ii) is complete. 
The exterior linear measure $l^{*}(S)$ of a set $S$ in the plane is defined as follows. Let $\varepsilon$ be a positive number, and let $F$ denote a sequence of (Euclidean) discs each having diameter less than $\varepsilon$ whose union contains $S$. Let $d(F)$ denote the sum of the diameters of the discs in $F$ (possibly $d(F)=\infty)$, let $l_{\varepsilon}(S)$ be the infimum of the numbers $d(F)$ for all such sequences $F$, and let $l^{*}(S)=\lim _{\varepsilon \rightarrow 0} l_{\varepsilon}(S)$. It is routine to prove that $l^{*}(S)$ is an outer measure in the sense of Carathéodory (see [11, p. 43]). Thus (see [11, remark on p. 48]), the analytic sets in $\Omega$ are linearly measurable (that is, measurable with respect to $l^{*}$ ). The same reference shows that analytic sets (in $\Omega$ or $C$ ) are Lebesgue measurable. Thus, since the Borel sets are analytic sets, we have the following corollary to Theorem 2.

Corollary. Suppose that $f$ is meromorphic in D. Then

(i)' for each Borel set $S \subset C$, both $\Gamma_{p}(S)$ and $\Gamma(S)$ are linearly measurable and Lebesgue measurable, and

(ii)' for each Borel set $S \subset \Omega, A(S)$ is Lebesgue measurable.

Remark. MacLane [7, Theorem 10] needed and proved (ii)' under the assumption that $f$ is holomorphic and $A$ is dense on $C$, and I extended MacLane's argument [9, Theorem 1] to prove that if $f$ is holomorphic and $S$ is a Borel set in $\Omega$, then $A(S)$ is a Borel set. These results were applied $\mathrm{n}$ the proofs of the theorems mentioned in Remark 3.

\section{Ambiguous properties}

The cluster set $C_{\tau}\left(=C_{\tau}(f)\right)$ of $f$ on the arc $\tau$ at $\zeta(\zeta \in C)$ is the set $\left\{a \in \Omega\right.$ : there exists a sequence $\left\{z_{n}\right\} \subset \tau$ such that $z_{n} \rightarrow \zeta$ and $\left.f\left(z_{n}\right) \rightarrow a\right\}$. We say that the point $\zeta$ is an ambiguous point of $f$ if there exist arcs $\tau_{1}$ and $\tau_{2}$ at $\zeta$ such that $C_{\tau_{1}} \cap C_{\tau_{2}}=\varnothing$. The fundamental result about ambiguous points is due to Bagemihl [1], and states that an arbitrary complex valued function in $D$ can have only countably many ambiguous points. Bagemihl, Piranian and Young [3] have introduced several types of ambiguous behavior, and have shown in particular that the modular function $m$, which maps $D$ onto the universal covering surface of $\Omega-\{0$, $1, \infty\}$, has the property that to each $\zeta \in C$ there correspond three arcs $\tau_{1}, \quad \tau_{2}$ and $\tau_{3}$ at $\zeta$ such that $C_{\tau_{1}}(m) \cap C_{\tau_{2}}(m) \cap C_{\tau_{3}}(m)=\varnothing$ (the threearc property at $\zeta$ ). The most nonambiguous behavior that $f$ can have at $\zeta$ $(\zeta \in C)$ is for there to exist $a \in \Omega$ such that for each arc $\tau$ at $\zeta, a \in C_{\tau}$; in this case we call $\zeta$ a principal point of $f$ ( $a$ is called a principal cluster value of $f$ at $\zeta)$, and we let $P(=P(f))$ denote the set of all principal points of $f$. In the case of the modular function $m, P(m)=\varnothing$; but we note that the ambiguous points of $m$ are dense on $C$. We prove 
Theorem 4. Suppose that $f$ is holomorphic in D, and suppose that $f$ has no ambiguous points in the open arc $\gamma$ of $C$. Then $P$ is residual on $\gamma$.

Proof. Let $U$ denote the union of all interiors of Koebe arcs of $f$ for the value $\infty$, and write $V=\gamma-\bar{U}$. Since $U$ is open, $\bar{U}-U$ is nowhere dense in $C$. Thus, since $U \subset P, \bar{U}-P$ is nowhere dense in $C$. Thus, in order to prove Theorem 4 , it is sufficient to prove that $V-P$ is a first category set. Let $\gamma_{0}$ denote one of the countably many components of the open set $V$, and note that we need only prove that $\gamma_{0}-P$ is a first category set. It follows from Theorem 1 that $A$ is dense on $\gamma_{0}$, and it is proved in [10, Corollary 1] under the assumption that $f$ is continuous in the extended sense and $A$ is dense on $\gamma_{0}$ that $P \cap \gamma_{0}$ is a $G_{\delta}$ set. Thus, in order to prove that $\gamma_{0}-P$ is a first category set, we need only prove that $\gamma_{0} \subset \bar{P}$. Suppose that this is not the case, and let $\gamma^{\prime}$ be an open subarc of $\gamma_{0}$ such that $P \cap \gamma^{\prime}=\varnothing$. If $A \cap \gamma^{\prime}$ has positive Lebesgue measure, it follows from Bagemihl's ambiguous-point theorem that some point of $A \cap \gamma^{\prime}$ is a principal point of $f$. Thus, from Theorem 1 we see that $\gamma^{\prime} \subset$ $\bar{A}(\{\infty\})$. Now let $\zeta \in \gamma^{\prime} \cap A(\{\infty\})$, and let $\tau$ be an arc at $\zeta$ on which $f$ has the limit $\infty$. Choose $0<\lambda_{n} \uparrow \infty$ such that each component of $\left\{z:|f(z)|=\lambda_{n}\right\}$ has no multiple points, and let $\Delta_{n}$ denote the component of $\left\{z:|f(z)|>\lambda_{n}\right\}$ that contains all points of $\tau$ that are sufficiently near $\zeta$. Since $\gamma^{\prime} \cap U=\varnothing$, for sufficiently large $n$ there is a component $\sigma_{n}$ of $\left\{z:|f(z)|=\lambda_{n}\right\}$ that is on the boundary of $\Delta_{n}$, separates $\Delta_{n}$ from the origin, and is such that $\bar{\sigma}_{n} \cap C \subset \gamma^{\prime}$. Since $\gamma^{\prime} \subset \bar{A}(\{\infty\})$, there exist points $\zeta_{n}^{1}$ and $\zeta_{n}^{2}$ in $\gamma^{\prime}$ such that $\sigma_{n} \cup\left\{\zeta_{n}^{1}, \zeta_{n}^{2}\right\}$ is a Jordan arc (or a Jordan curve); and since $\zeta$ is not an ambiguous point of $f, \zeta_{n}^{j} \neq \zeta(j=1,2)$. Thus, $\zeta_{n}^{1}$ and $\zeta_{n}^{2}$ are in different components of $\gamma^{\prime}-\{\zeta\}$. It is clear then that $\infty$ is a principal cluster value of $f$ at $\zeta$, and with this contradiction, we see that the proof of Theorem 4 is complete.

F. Bagemihl suggested to me that the Schwarzean triangle functions should serve to prove

Theorem 5. There exists a function $S$ meromorphic in $D$ that has no ambiguous points, and has the three-arc property at each point of $C$. In particular, $P(S)=\varnothing$.

Proof. Define the Schwarzean triangle function $S$ as follows. Let $\Delta$ be a noneuclidean (hyperbolic) triangle in $D(\bar{\Delta} \subset D)$ with angles all having magnitude $\frac{\pi}{4}$, and let $S$ map $\Delta$ conformally onto the upper halfplane so that the vertices correspond to the points 0,1 and $\infty$. It is well known (see [5, pp. 158-162]) that we can, by repeated reflection, extend $S$ on $\Delta$ to a function $S$ meromorphic in all of $D$. It is clear that $S$ has the three-arc property at each point of $C$. We prove that $S$ has no ambiguous points by proving the stronger statement: for any two boundary curves $\alpha$ 
and $\beta, C_{\alpha}(S) \cap C_{\beta}(S) \neq \varnothing$. Suppose that this is not the case, and let $\alpha$ and $\beta$ be boundary curves such that $C_{\alpha}(S) \cap C_{\beta}(S)=\varnothing$. Then, since $C_{\alpha}(S)$ and $C_{\beta}(S)$ are compact in $\Omega$, there exists a Jordan curve $J$ that does not contain 0,1 or $\infty$ and is such that $C_{\alpha}(S)$ and $C_{\beta}(S)$ are contained in different components of $\Omega-J$. Let $U$ denote the component of $\Omega-J$ that contains at most one of the points 0,1 and $\infty$, and suppose that the notation is such that $C_{\alpha}(S) \subset U$. Since $U$ contains at most one of the points 0,1 and $\infty$, each component of $S^{-1}(U)$ has positive distance from $C$; and since $C_{\alpha}(S) \subset U, \alpha$ is eventually (for sufficiently large $|z|)$ in one component of $S^{-1}(U)$. Thus, we have a contradiction, and the proof of Theorem 5 is complete.

University of Wisconsin-Milwaukee 


\section{References}

[1] Bagemthl, F.: Curvilinear cluster sets of arbitrary functions - Proc. Nat. Acad. Sci. U.S.A. 41 (1955), $379-382$.

[2] -»- Sets of asymptotic values of positive linear measure - Ann. Acad. Sci. Fenn. A. I. 373 (1965), 1-7.

[3] -»- Piranian, G., and Young, G. S.: Intersections of cluster sets - Bul. Inst. Politehn. Iasi. N.S. vol. 5 - No. 9 (1959), $29-34$.

[4] -"- and Seides, W.: Koebe ares and Fatou points of normal functions Comment. Math. Helv. 36 (1962), 9-18.

[5] Carathéodory, C.: Funktionentheorie II. - Basel, 1950.

[6] Collingwood, E. F., and Cartwright, M. L.: Boundary theorems for functions meromorphic in the unit circle - Acta Math. 87 (1952), 83-146.

[7] Maclane, G. R.: Asymptotic values of holomorphic functions - Rice Univ. Studies vol. 49 - No. 1, Winter 1963.

[8] Mazurkiewicz, S.: Sur les points singuliers d'une fonction analytique - Fund. Math. 17 (1931), 26-29.

[9] McMrluan, J. E.: Asymptotic values of functions holomorphic in the unit disc Michigan Math. J. 12 (1965), 141-154.

[10] -》- Principal cluster values of continuous functions - Math. Z. (to appear). [11] SAKs, S.: Theory of the Integral, second revised edition - New York, 1937.

[12] SeIBert, P.: Über die Randstructuren von Überlagerungsflächen - Math. Nachr. 19 (1958), 339-352.

[13] Sierpinski, W.: General Topology - Toronto, 1952. 